\title{
IMPORTANCIA DEL BROMOFORMO EN CIERTAS
} INVESTIGACIONES METALURGICAS

\author{
PO EDUARDO SCHMITTER
}

Trabajo presentado-en la Seaión Ordjn natia de la Sociedad Geológiea Mesicans, el 3 de abril de 1936.

Bien conocido es en los estudi-os de petrografía sedimentaria el uso del bromoformo, pues por su fácil manejo, recuperación y su acción casi nula sobre los distintos constituyentes mineralógicos de un sedimento, es inmejorable para separar minerales con densidades mayores y menores de 2.9 cuando se le utíliza puro.

Isómero del cloroformo, se deriva del metano por sustitución de tres átomos de hiarrógeno con los correspondientes de bromo, eonstituyéndose en tri-bromo-metano o bromoformo.

Su densidad es de 2.90350 a $15^{\circ} \mathrm{C}$. en relación con la del agua a $4^{\circ} \mathrm{C}$.

Su punto de ebullición a presión normal es de $149.55^{\circ} \mathrm{C}$. 11).

Diluido en las debidas proporciones con benzeno o tetracloruro de carbono proporciona una serie de "líguidos pesados" cuyas densidades tienen por límite inferior las de los compuestos indicsdos, expresados a continuación:

Benzeno.-Densidad a $25^{\circ} \mathrm{C}$. en relación con la del agua a $4^{\circ} \mathrm{C} .=87369$. Punto de ebullición a presión normal $=$ SO. $122^{\circ} \mathrm{C}$.

(1) Datos tomados de "Ohemical Engineer's Handbook" ge H. PeTTy, 1a; ed. p. 281, 1984. 
Tetracloruro de carbono.-Densidad a $25^{\circ} \mathrm{C}$. en relación con la del agua a $4^{\circ} \mathrm{C} .=1.5844$. Punto de ebullición a presión normaI $=76.75^{\circ} \mathrm{C}$. (2).

Teniendo en cuenta que una gran parte de los problemas metalúrgicos se relacionan con el beneficio de menas metálicas pobres, he creido de alguna utilidad recomendar el uso del bromoformo en los estudios preliminares de laborato. rio, cuando de apluellas se trate.

$\mathrm{Su}$ aplicación en este caso se encuentra en intima relación con el análișis mecánico de los minerales y su objeto es la concentración prácticamente cuantitativa de los que tie. nen densidades mayores de 2.9. Esto se logra fácilmente si se tiene en cuenta que las "matrices" más comunes constituí. das por cuarzo, feldespatos, calcita, dolomita, etc., tienen sus densidades menores de 2.9 .

El proceso general que se sigue en el laboratorio para estudiar una mena después de un muestreo bien hecho, se continúa con examen preliminar al microscopio para deter. minar la constitución mineralógica, las relaciones genéticas de los minerales, su estado de alteración, el tamaño y forma de los granos, la textura de la mena, etc. Hechas tales determinaciones, se obtienen un conjunto de datos, en parte definitivos y en parte requiriendo la contribución de las siguientes etapas -del proceso de investigación.

En seguida una parte de la muestra molida en condiciores prácticas, se somete a una clasificación por tamices que ,permitirá la separación por tamaños de las partículas.

En este punto una conv-eniente separación con bromo. formo de minerales "ligeros" y "pesados" en cada tamaño, nos indicará el grado de molienda a que deberá llegarse para poner en Tibertad la mayor parte de las partículas de minerales por concentrar.

Si los metales por beneficiar fueran entre otros el oro y la plata, procede practicar en 3 porciones de la parte ligera: gruesa, media y fina, un ensaye por los mismos metales pa-

(2). Igual referencia que en la nota (1). 
ia determinar si la matriz lleva una parte de éstos en par. tículas tan pequeñas que no fueron suficientes para influenciar notablemente la densidad de las particulas "ligeras", en cuyo caso tales valores resultarian difieilmente recuperables por concentración. Con las porciones correspondientes a los minerales "pesados" se preparan briquetss de baquelita, las que una vez pulidas, se someterán al examen microscópico pa. ra determinar un segundo grado de molienda si se hiciera recesaria la flotación selectiva de 2 o más minerales "pesa. dos", que estuvieran mezclados íntimamente.

Una vez alcanzada esta etapa en la investigación con la contribución indispenşable del análisis químico, el resto de la muestra se encuentra en condiciones de ser tratada en las máquinas concentradoras, teniendo en cuenta todos los datos anteriores.

Si la molienda recomendada por la primera clasificación "no fué suficiente para poner en libertad los minerales por con. centrar, es necesario practicar una segunda clasificación por tamices, seguida de un tratamiento con bromoformo como que. da indicado, en una porsión de las "colas" procedentes de las máruinas concentradoras.

El modo de operar con el bromoformo es el siguiente: cada porción procedente de la clasificación por tamices, se $\mathrm{v}$ colocando en el aparato separsdor .(fig. 1), que consiste simplemente de un embudo fijo en soporte de fierro con capacidad suficiente para contener la porción y seis veces su volumen de bromo-forDlQ. Dicho embudo obturado previamente por la pa,rte interior con un tapón de corcho de la forma indicada en la (fig. 1), Y manejable por un mango de vidrio suficientemente largo, incrustado en dicho tapón. (3).

En tales condiciones se vierte el bromoformo en el embudo, llenando la capacidad restante, se agita lenta y constantemente durante unos 10 minutos, al cabo de los cuales

(3). Henry B. Miner en su tratado de petrografia sedimentaria, 2a: edición, de 1929, pág. 43, iJustrs ol misno apsrato pero con obtu. tación en el pico del embudo, mediante un pequeño tubo de hule cerra. do con una pinzs de Mohr. Este arreglo no me parece conveniente, en vista de que el bromoforno puede actuar como disolvente del bole, 


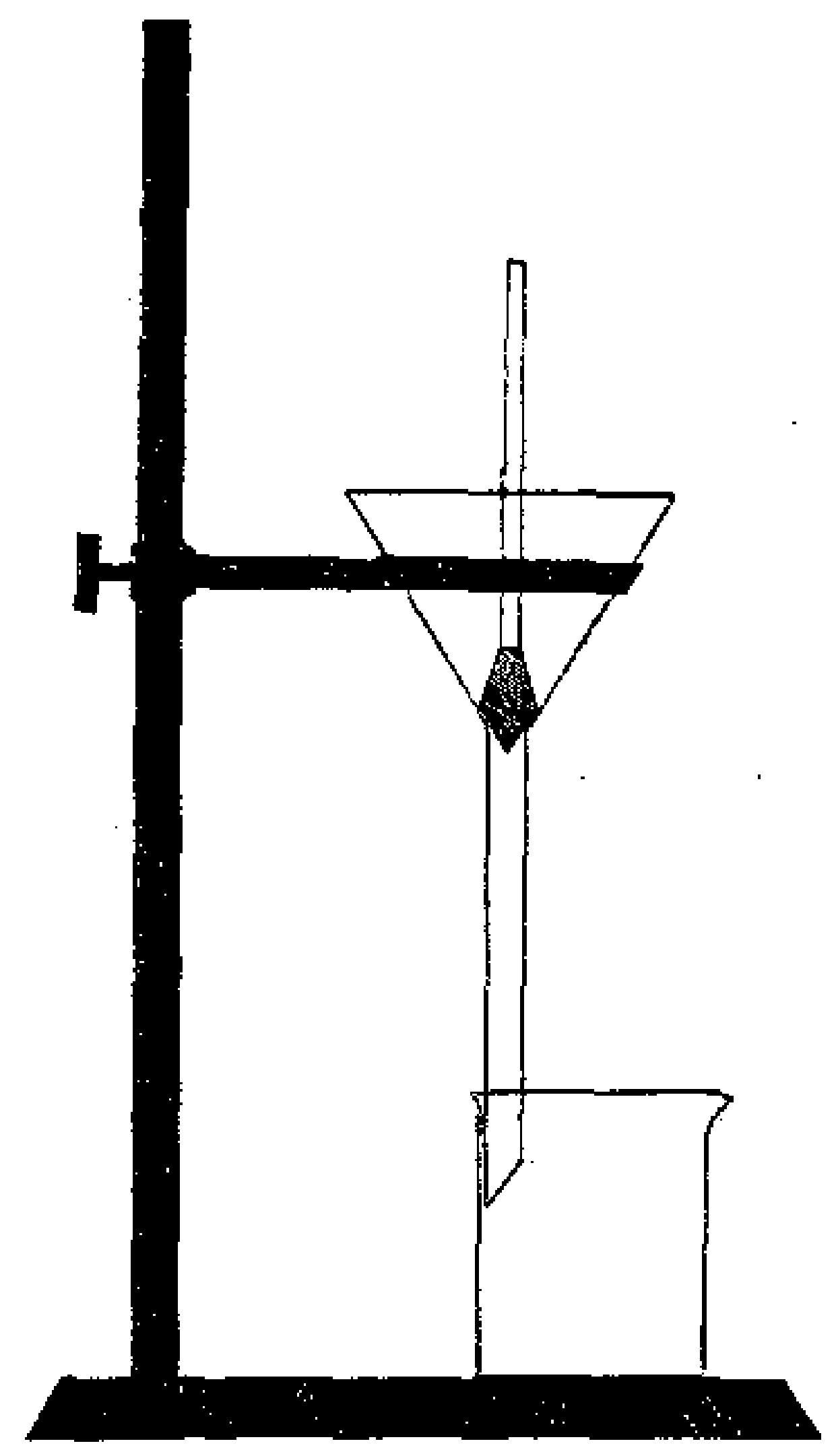

Figura Núm. 1.

se suspende la agitación. Entonces los minerales con densidad mayor de 2.9 quedarán en el fondo del embudo perfectamente separados de los ligeros, que flotarán eñ la superficie dei líquido.

Colocando un vaso de precipitados de suficiente capacidad abajo del embudo, se procede a levantar -el tapón durante un instante con un movimiento violento, para dejar pasar todas las partículas de mineraJes "pesados", después de 10 cual se procede a la obturación con igual impulso.

Hec'ha la operación, los minel'ales "pesados" contenidos en el fondo del vaso, se separan de la cantidad de bromoformo que salió junto con ellos, por deca.ntacióll. 
En el embudo que contiene la mayor parte del bromofor: roo con los minerales "Iigeros", se levanta el tapón de marera que sólo pase el bromoforrno lo más violento posible a otro vaso previamente colocado debajo del embudo. Una vez libres las dos porciones de bromoformo. se guardan en su frasco que deberá ser de vidrio color ámbar y con tapón esmedIado. En vista de la pérdida sensible de bromaformo pOl' volatilización, $\mathrm{y}$ de su posible combinación con algunas partículas minerales, es necesario separarlo de las mismas y guardarlo lo más pronto posible.

Los minerales que- aûn se encuentran mojados con bro moformo y reteniendo una parte de éste, se lavan a continua. ción un suficjente número de veces con benzeno o tetracloru. ro de corbona,

Todas las porciones de lavado se guardan en un frasco para efectuar en su oportunidad, la recuperación del bromo. formo pOr destilación fraccionada, mediante un aparato como el indicado en la figura número 2 .

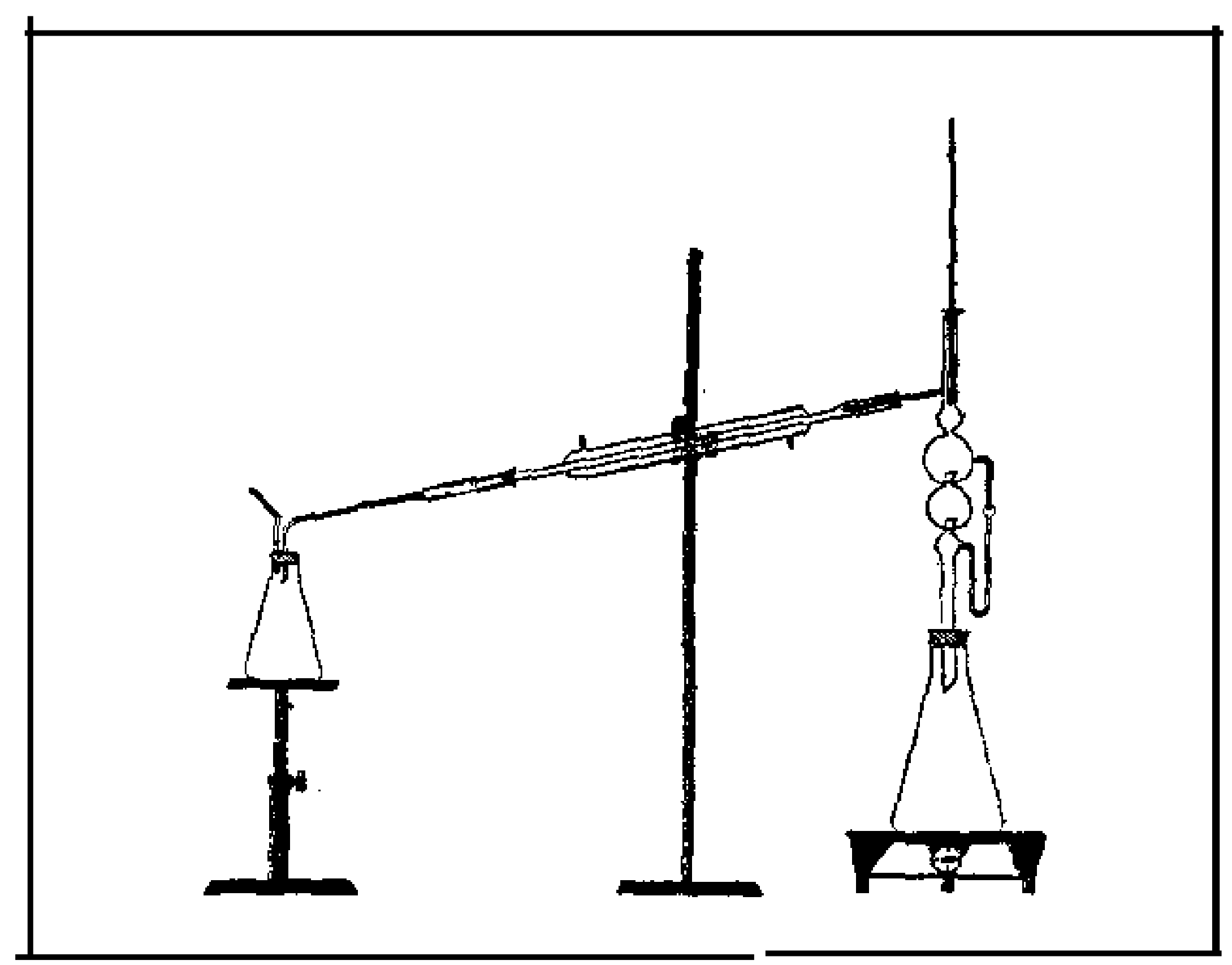

Fig. Núm. 2. 
Este consta en sus partes esenciales de un matraz de destilación, un rectificador, un termómetro con escala de $200^{\circ} \mathrm{C}$, un refrigerante y un matraz para recibir los productos de la destiladón. Las obturaciones se hacen con tapones de corcho y todo el aparato se fija en un soporte de fierro mediante pinzas "universales". La instalación se completa con un elemento eléctrico de 3 calores para el calentamiento del matraz.

A continuación cito algunos de los problemas prácticos en los que utilicé con éxito el método indicado:

1.-En una muestra de arenas auríferas se trataba de averiguar cuantitativamente el tamaño, aspecto y la forma de las partículas de oro con el objeto de recomendar un método apropiado de beneficio. Como el dato debía ser cuantitativo tuve que desechar el método de "tentadura" y aplicar el del bromoforrno con examen al micrascopio.

La muestra estaba formada por los munerales siguientes en orden aproximado de abundancia: pirita. cuarzo. feldespatos, magnetita, minerales pesados no magnéticos y oro Jibre. Separado cada tipo de mineral después de la clasificación por tamices. por medio del bromoformo, un imán, atague químico y nitrato de talio, se anotaron las cantidades en porciento que de cada uno habia y se expresaron en gráfica, cuya interpretación, indicó la derivación de las partículas de oro en compañía de.las de pirita. dato complementario de cierta importancia para localizar en el arroyo la presencia de los valores, en los puntos en donde se notara concentración de aquel sulfuro que en este caso particular. s'e presentó en la forma de pequeños piritoedros de tamaños uniformes entre cortos lío mites de fineza. Separado el oro se pudo examinar fácilmente al microscopio para completar la investigación.

2._Estudiando la posibilidad de concentrar los valores en oro de una muestra de jales cuya composición mineraló. gica principal. consistía de cuarzo, hematita y oro, se trató dé averiguar el tamaño de las partículas y con cual de los dos minerales se encontraba asociado. No habiendo podido preparar briquetas " para examinarlas al microscopio, se tra- 
t6 la muestra con bromoformo después de la consabida clasificación. Practicados los ensayes en cada porción de los mi. 11 crales "ligeros" y "pesados", la ley de 14 gramos por tonelada fué sensiblemente la misma para todos, comprobando con ésto, que el oro se encontraba en partículas muy pequeñaz y con igual distribución en los minerales indicados. Por tanto, no se pudo recomendar ningún método de concentraci6n.

3.-Con objeto de ensayar un método de análisis por berilium en una muestra de cuarzo con berilo procedente de la 'Sierra de la Madera en Sonora, separé fácilmente los dos minerales mediante una molienda relativamente gruesa, seguida de tratamiento Con hromoformo' en tetrae1oruro de carbono, solución con los constituyentes en tales proporciones, que dió una densidad de 2.67. El cuarzo flotó quedando el berilo en el fondo del embudo.

4.-Tratando de averilguar las posibilidades de concentración por flotación en una muestra compuesta en su mayor parte de hematita, cuarzo y límonita con muy pequeña pro)lorción de pirita y calcopirita, con ley de 180 y 28 gramos llOr tonelada de plata, y oro respectivamente, se practic6 la investigación con el muestreo y examen mirroscópico seguidos de una clasificación por tamices, ensayándose por oro $\mathrm{Y}$ plata, parte de cada una de las prociones, anotando gráficamente los resultados.

A continuación se tomaron los sobrantes de la clasificación, se,pesó una cantidad igual de cada po,rción y se trató con bromoformo. Calculados los porcentajes de minerales "pesados" contenidos en cada porción de la muestra clasificada, se anotaron gráficamente. Entonces al estudiar las curvas se notó (fig. 3), que la correspondiente a las leyes de oro y la de los \% indicados resultaron muy semejantes, y diferentes notablemente de la curva correspondiente a las leyes de plata.

Esto demostró la íntima relación de la hematita con el cro, que indudablemente se encontraba en partículas pequeñas, uniformemente repartidas dentro de aquélla y sensiblemente de igual tamaño. Por tanto, al efectuar las prue. 


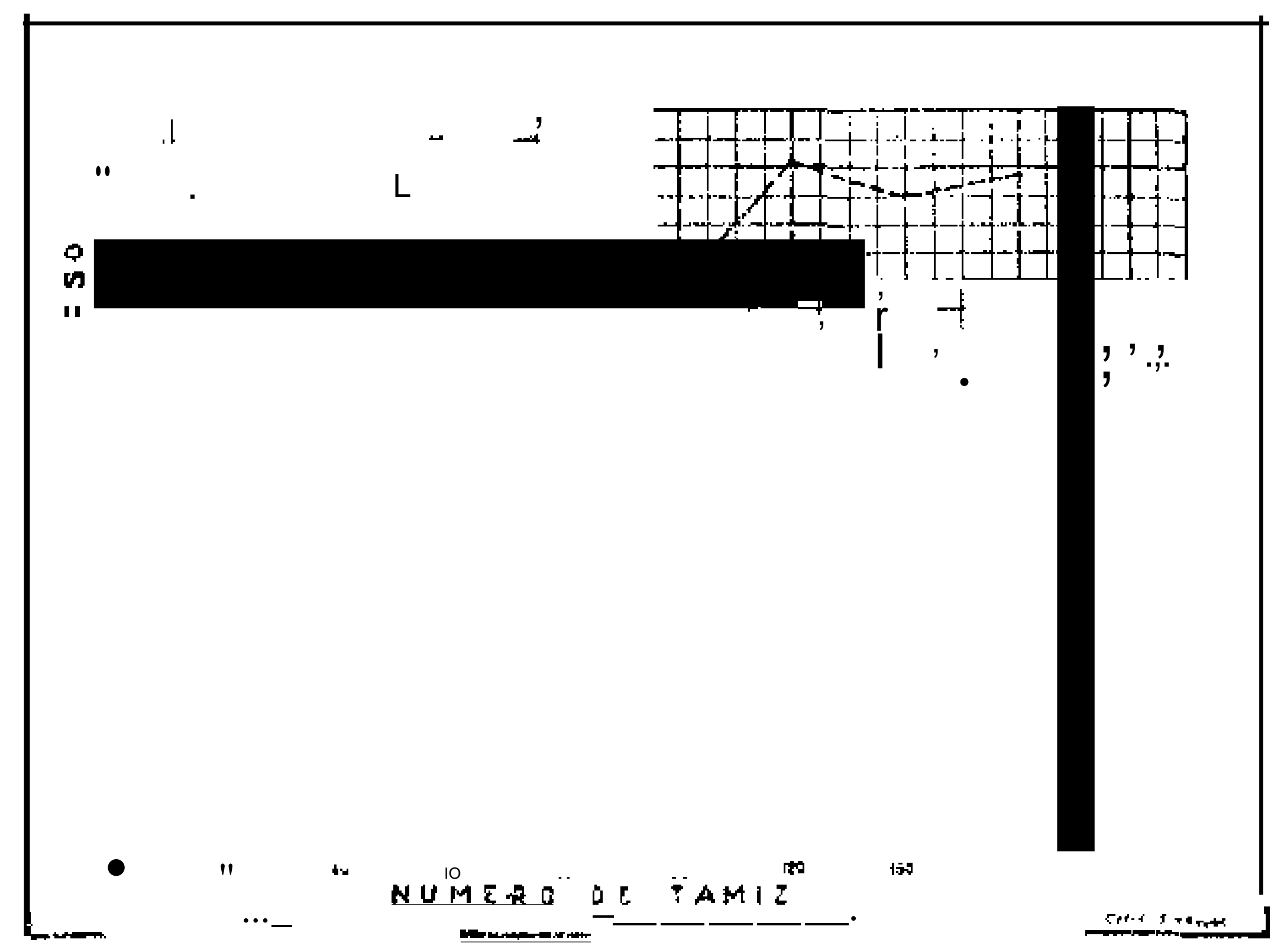

Fig. Núm. 3.

bas de flotación. nQ se obtuvo una extración en oro arriba dcl 76\% a causa de la interferencia presentada por la hematita.

Por otra parte, la diferencia de las dos primeras con la de las leyes de plata, nos indicó la falta de asociación de ésta con el oro.

5.-Un kilogramo de arena de río fué tratado para investigar su contenido de casiterita y la posible concentración de dicho mineral.

Se practicó una clasificación por tamices que no se pudo interpretar, por no indicar el análisis Químico más que huellas probables de estaño. En seguida se dividió la muestra en dos porciones mediante un tamiznúnIero 20. La porción más gruesa se trató en máquina pulsainte para efectuar la concentración de los valares y la más fina, se trató en mesa concentradora con el mismo objeto.

Examinados al microscopio cada uno de los productos obtenidos, "concentrados", "medios" y "colas", se encontró 
una composición mineráalógica más a menos constante, con ljgeta variación en las partículas más finas.

Una ver anotados los pesos obtenidos de cada porción, y en vista del mal resultado que habia tenirlD la primera clasifjecación pOr tamices y la concentración en las máquinas, se procedió a tratar cada producto con bromoformo, encontrando (IUe la mesa habia efectuado la mejor concentración de minerales "pesados". Una vez anotados los pesos de minerales "ligeros" y "pesados" para calcular la concentración y rendimiento obtenidos, se juntaron todas las porciones de "ligeros" y "pesados' por separado, practicándo a continuación lma nueva clasificación por tamices con cada una de las dos porciones resultantes.

Se calculó entonces el porcentaje total de los dos tipos de minerales y el porcentaje de "pesados", conteniclos en cada porción de los "ligeros"; se anotaron los resultados gráficamente y de la interpretación de lass 3 curvas se dedujo 10 siguiente: (fig. 4).

lo.-Los diámetros de partícujas comprendidos entre '.104 y .295 mm., resultaron ser los límites más prácticos de fineza para beneticiar las arenas.

20. En cuanto a las condiciones de acarreo que prevalecieron corriente arriba del punto en donde se tomó la muestra, puede decirse que sólo las. partículas de minerales "ligeros" y "pesados" que tuvierQn diámetros aproximados entre .295 Y .074 mm. (límite de fineza experimentada), estuvieron en condiciones de movimiento simultáneo para dar oportunidad a las partículas "pesndas", de concentrarse en el lugar muestreado.

Por otra parte, hecho el ensaye de estaño sobre $.5 \mathrm{gra}$ mos del total de minerales "pesados" y el de oro y plata so bre 5 gramos, después de practicar las operaciones anteriores, los resultados fueron los siguientes:

$$
\begin{aligned}
& \mathrm{Sn}=.006 \% \\
& \mathrm{Au}=.041 \text { gramos por tonelada } \\
& \mathrm{Ag}=.39 " \mathrm{n} "
\end{aligned}
$$




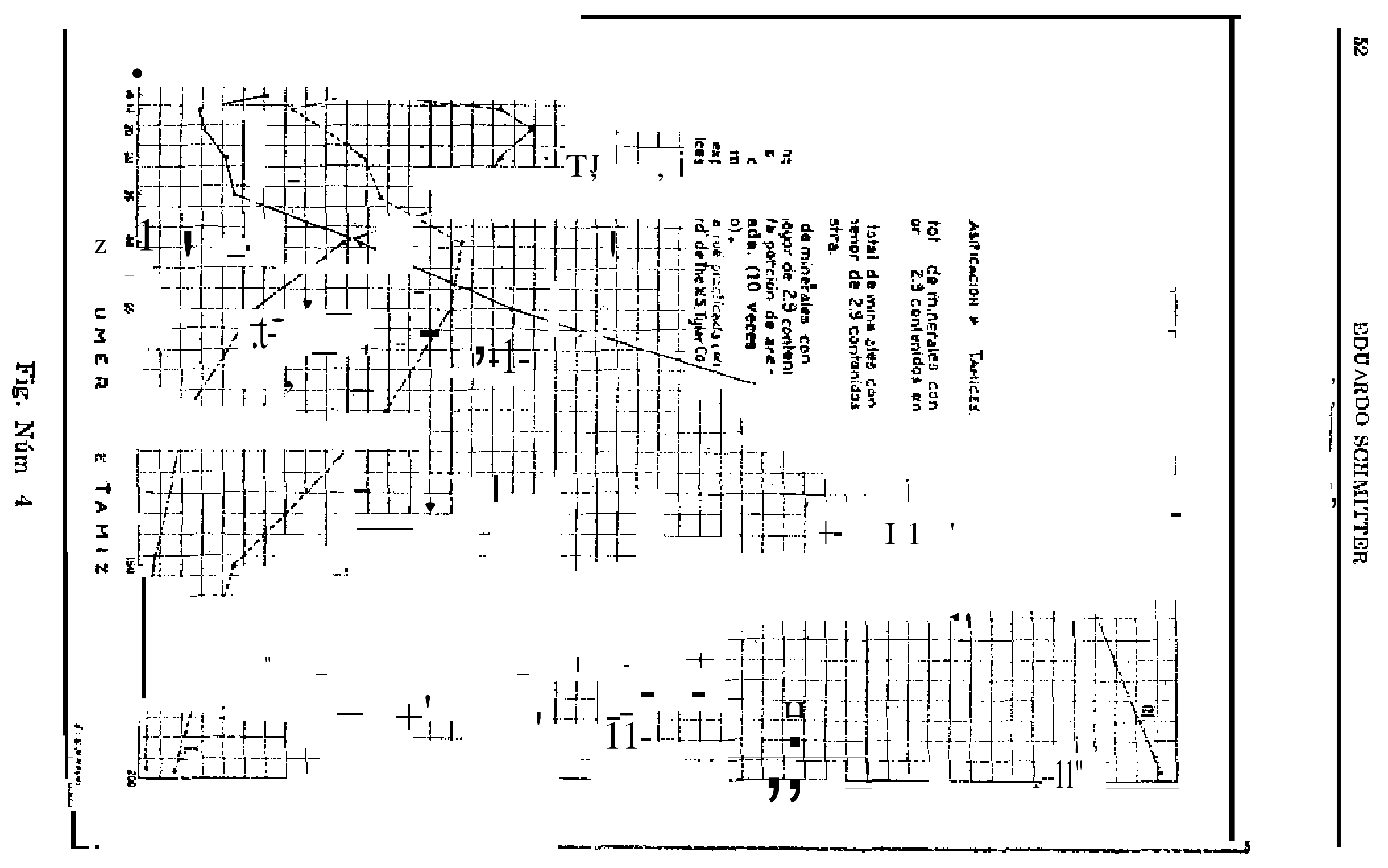


Leyes de oro y plata que para ontener'se por ensaye ordimario, hubiera sido indispensable practiesr alrededor" de 100 fusiones de 10 gramos cada una, yel mismo número de determinaciones por Sh suponiendo que en cada determinación se hubieran atacado 5 gramos de muestra.

Es claro que con semejantes resultados el estudio de la muestra fué inútil para aplicarse al beneficio de los metajes ensayados; pero no hubiera sido así, en el caso' de haber te. nido que investigar platino, elementos radioactivos o elementos raros en general, cuya focalización es de bastante importancia; por eso cité este último caso en el que como en los anteriores, la contribución de\} bromoformo ayudó a la resolnción del problenar.

México, D. F., a 3 de abril d'e 1936.

Eduardo SCHMITTER. 